\title{
Role of the oocyte nucleus in determination of the dorsoventral polarity of Drosophila as revealed by molecular analysis of the K10 gene
}

\author{
Edouard Prost ${ }^{1,4}$ François Deryckere, ${ }^{4}$ Christophe Roos, ${ }^{2}$ Marc Haenlin, ${ }^{3}$ Véronique Pantesco, and \\ Eliane Mohier ${ }^{5}$ \\ Laboratoire de Génétique Moléculaire des Eucaryotes du CNRS, Unité 184 de Biologie Moléculaire et de Génie Génétique de \\ l'INSERM, Faculté de Médecine, 67085 Strasbourg Cédex, France
}

\begin{abstract}
In Drosophila, the establishment of dorsoventral polarity of the developing embryo depends on the expression of at least 11 maternally acting genes. Mutant females that lack any of these gene activities produce normally shaped eggs that develop into dorsalized embryos. The female sterile $K 10$ mutation differs from these mutants, because in addition to the dorsalized development of the embryo, it causes a dorsalization of the egg shape. During oogenesis, the $\mathrm{K1O}$ gene is specifically expressed in the oocyte. Antibodies raised against a $\beta$ galactosidase-K10 fusion protein were used to visualize the $K 10$ product in ovaries by indirect immunofluorescence. The protein, which contains a putative DNA recognition helix, accumulates in the nucleus of the oocyte, where it is assumed to have a regulatory function. Our results thus indicate that the controlled expression of some of the genes of the oocyte nucleus is essential for the determination of the dorsoventral polarity of the oocyte and possibly of the developing embryo.
\end{abstract}

[Key Words: Drosophila; dorsoventral; oocyte; K10 gene]

Received March 14, 1988; revised version accepted May 12, 1988.

One of the goals of developmental biology is to understand the mechanisms underlying embryonic pattern formation. In Drosophila, the identification and genetic analysis of mutations affecting embryonic development have shown that the first step in the formation of the spatial pattern of the embryo is the definition of the two primary axes: the anterior-posterior axis and the dorsoventral axis (Nüsslein-Volhard 1979). Dorsoventral polarity is already apparent in the shape of the wild-type egg at the time it is laid. The egg has a curved ventral side and a flattened dorsal side, with two chorionic appendages implanted dorsally (Fig. 1). The second visible manifestation of dorsoventral polarity arises at gastrulation, when the ventrally located cells of the cellular blastoderm invaginate to form the ventral furrow.

So far, 11 maternally expressed loci have been identified, the products of which are required for the establishment of the dorsoventral pattern (Anderson and Nüsslein-Volhard 1984a,b, 1986). The loss of any of these gene products by mutation leads to a common recessive phenotype initially described for the prototype of

Present addresses: 'Institut Pasteur, 75015 Paris, France, ${ }^{2}$ Department of Genetics and Recombinant DNA Laboratory, University of Helsinki, Finland; ' ${ }^{3}$ stitut für Entwicklungsphysiologie der Universităt, $5000 \mathrm{Co}$ logne 41, Federal Republic of Germany.

"E.P. and F.D. are to be considered as equal first authors.

${ }^{5}$ Corresponding author. these mutations: dorsal (dl). Females homozygous for a dorsal-like mutation lay normally shaped eggs that develop in such a way that dorsal pattern elements are formed at the expense of lateral-ventral pattern elements. It has been proposed that each of these dorsalgroup gene products is necessary for generating a gradient of positional information along the dorsoventral axis of the cellular blastoderm (Anderson et al. 1985a,b). However attractive and credible this model may be, it does not predict what the initial localized cue required for triggering the later processes may be, nor does it explain how this gradient is generated relative to the polarity of the egg.

To trace the origin of this egg polarity and to investigate how it might further influence the polarity of the developing embryo, it was of interest to analyze mutations affecting the polarity of both the egg and the embryo. The female sterility mutation $K 10[f s(1) K 10]$ satisfies both of these criteria (Wieschaus et al. 1978; Wieschaus 1979, 1980). Females homozygous for K10 lay eggs that are almost cylindrical. The dorsal appendages are fused by material encircling the egg, as if its entire circumference had become dorsalized. The dorsalization of $K 10$ oocytes can already be detected during oogenesis, at a time when normal ovaries show a clear dorsoventral polarity (stage 11 of King 1970). The wild-type oocyte 
nucleus is acentric and lies near the surface at the anterior side of the oocyte (Fig. 1). Due to an asymmetric migration, the overlaying follicle cells become much thicker in this region than those on the opposite side of the oocyte. This region, where the dorsal appendages will be formed, corresponds to the prospective dorsal side of the embryo. Although the oocyte nucleus is still acentric in $K 10$ egg chambers, all of the follicle cells surrounding the anterior side of the oocyte show the thickened appearance normally found at the dorsal side.

In spite of this effect on the follicle cells and on the chorion they secrete, Wieschaus et al. (1978) showed that the K10 mutation is strictly germ-line dependent, suggesting that the follicle cells are most likely instructed by positional cues provided by the developing oocyte or by the nurse cells. When fertilized $(1$ or $2 \%)$, K10 eggs show the dorsalized development that is common to the dorsal-like mutations.

The fact that the $K 10$ mutation, as well as two others recently described by Schupbach (1987), affects both the polarity of the egg shape and the polarity of the developing embryo demonstrates that there is a time during development when both polarities are coupled.

The $K 10$ gene has been cloned and its function assigned to a 5-kb DNA fragment by P-element transformation. This fragment encodes a major transcript of 3.1 $\mathrm{kb}$, which is specifically transcribed in the oocyte (Haenlin et al. 1985, 1987).

To understand the role of the $K 10$ product in the establishment of dorsoventral polarity, we have raised polyclonal antibodies specific for $\beta$-galactosidase $-K 10 \mathrm{fu}$ sion protein, which permitted the visualization of the $K 10$ product on ovary sections by indirect immunofluorescence. We show that the product accumulates in the oocyte nucleus. We also report the nucleotide sequence and the putative protein sequence of K10. Although it does not contain a homeo box, the sequence encodes a putative DNA recognition helix. Together, the nuclear localization of the protein and the putative DNAbinding motif suggest that $K 10$ might possess a regulatory function.

\section{Results}

\section{Molecular structure of $\mathrm{K} 10$ mRNAs}

The $K 10$ gene was cloned by the microdissection technique and positively identified by germ-line transformation (Haenlin et al. 1985). It was localized to a 5-kb DNA fragment, the transcriptional organization of which is summarized in Figure 2. A major transcript of $3.1 \mathrm{~kb}$ is abundant in ovaries and early developing embryos and is likely responsible for the $K 10$ function. Using the $5^{\prime}$ HindIII-EcoRI and the $3^{\prime}$ EcoRI-SalI genomic fragments, we screened cDNA libraries. From pools of 0 - to 3- and 3- to 6-hr embryos, we isolated $>30$ clones, three of which are represented in Figure $2 \mathrm{C}$ (Haenlin et al. 1987).

A more detailed description of the $K 10$ gene was obtained by sequencing (Sanger et al. 1977; Lin et al. 1985) the $5^{\prime}$ region of cDNA EG15 and the entire $1.5 \mathrm{~kb}$ of
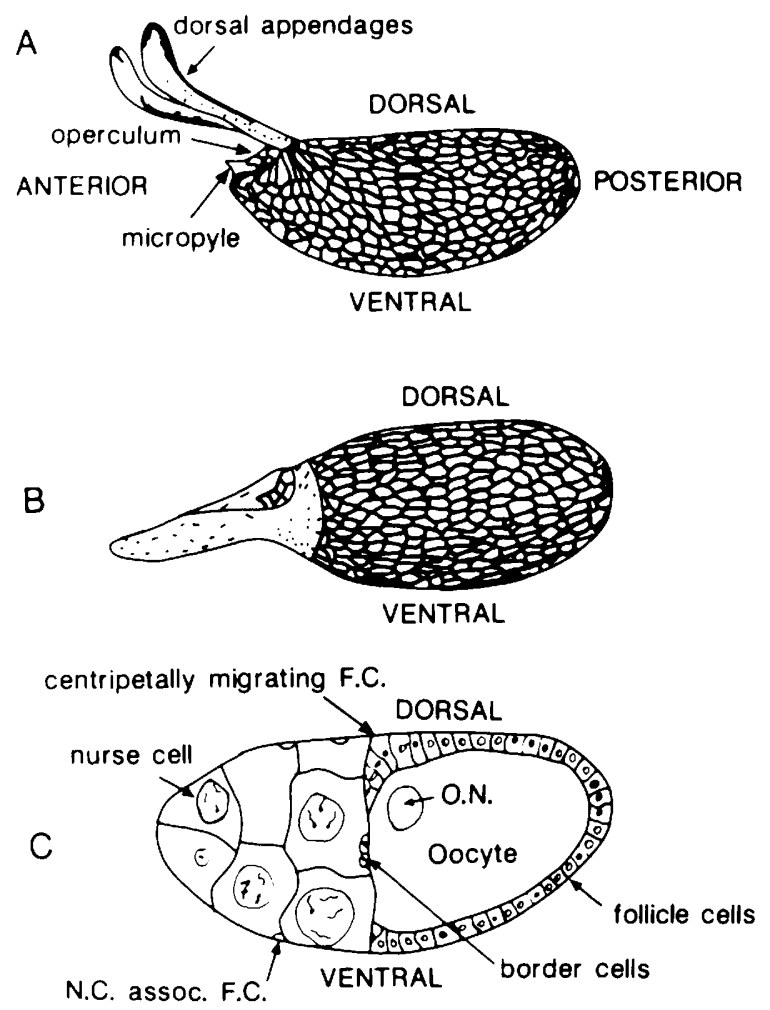

Figure 1. (A) A mature wild-type Drosophila egg, illustrating the anterior-posterior and dorsoventral polarity landmarks of the shell. The two respiratory appendages are implanted at the anterior-dorsal end of the egg, immediately above the operculum. The micropyle, a small cone used for sperm entry, protrudes from the mid-ventral part of the operculum. The net that covers the whole egg circumference corresponds to the imprints left by the follicle cells, which secrete the chorion during the last stages of oogenesis. (B) A mature $K 10$ egg. The appendages are fused at their base by material completely encircling the operculum, leaving only a small access to the micropyle; this explains the low rate of fertilization of these eggs $(1-2 \%)$. Note that in addition to the roughly cylindrical egg shape, the pattern of follicle cell imprints in the lateral and ventral regions are more elongated than normal, reminiscent of those found in the dorsal side of the wild-type egg. $(C)$ Section through a stage11 egg chamber. Criteria for staging differ among investigators. For our purposes, the chronology of the events taking place around this stage is as follows: Stage 10A, the transition between columnar follicle cells covering the oocyte and the squamous follicle cells covering the nurse cells becomes abrupt; stage 10B, follicle cells begin centripetal migration between the oocyte and the nurse cells; stage 11, follicular epithelium thickens in the region adjacent to the oocyte nucleus, which also corresponds to the region where the appendages form /stage $12)$. The oocyte nucleus $(O . N$.) occupies an anterior acentric position as early as stage 8 , i.e., at the beginning of vitellogenesis. $K 10$ egg chambers show a similar appearance until stage 11 , when follicle cells start their centripetal migration. Although the oocyte nucleus is still acentric, the follicular epithelium has a thickened appearance all over the anterior end of the oocyte (observations not shown).

cDNA E51, as well as portions of the 6-kb genomic DNA segment that include the entire transcribed region /Fig. 


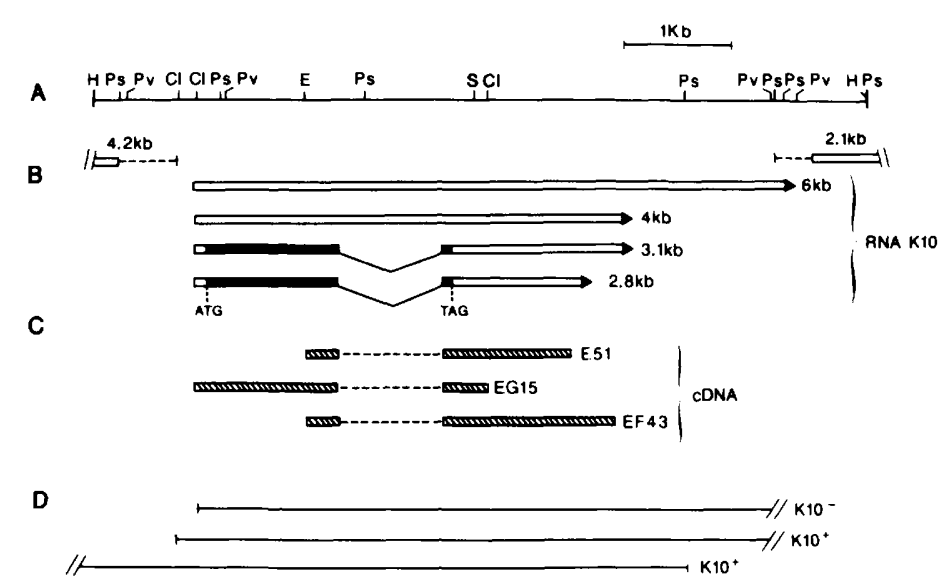

Figure 2. Transcriptional organization of the $K 10$ gene. $(A)$ Restriction map of the K10 locus. $(\mathrm{H})$ HindIII, (Ps) PstI, (Pv) PvuII, (Cl) ClaI, (E) EcoRI, (S) SalI. (B) Localization of the transcripts, as defined by Northern blot analysis. The 4.2- and the 2.1-kb RNAs that map on either side of $K 10$ correspond to the $\mathrm{kz}$ and $\mathrm{cm}$ loci, respectively. The open reading frame for the $3.1-$ and the $2.8-\mathrm{kb}$ transcripts is shown by filled boxes. $(C)$ Structures of the cDNA clones used in this analysis. cDNA libraries were kindly provided by L. Kauwar, S. Artavanis-Tsakonas, and H. Jäckle. Hatched boxes represent exonic regions. $(D)$ Positioning of the $K 10$ function by P-element-mediated transformation [for details, see Haenlin et al. 1987]. The construction starting at the ClaI site is still capable of restoring the $K 10$ function.
3). Comparison of the genomic and cDNA sequences confirms that a single intron, $854 \mathrm{bp}$ long, is spliced out of the mature transcript. The $5^{\prime}$ end of the major transcript, not present on either of the cDNA clones, is inferred from S1 mapping and primer extension experiments (data not shown) and is found $\sim 40$ bp upstream from the $5^{\prime}$ end of cDNA EG15. This promoter region is likely to be shared by all transcripts, as suggested by hybridization of poly $(\mathrm{A})^{+}$RNA Northern blots, with small probes originating from the $5^{\prime}$ end of the transcribed region (Haenlin et al. 1987). This low abundance of the minor transcripts did not permit confirmation of their common $5^{\prime}$ end, either by S1 mapping or by primer extension experiments. The region immediately preceding the transcriptional start site contains no canonical TATA box sequence. A consensus polyadenylation signal (AATAAA) occurs about $20 \mathrm{bp}$ upstream of the mapped $3^{\prime}$ end of the poly(A) tail of cDNA EF43.

\section{The predicted $\mathrm{K} 10$ protein}

The sequence analysis reveals a long open reading frame encoding a putative protein of 463 amino acids, $51.5 \mathrm{kD}$, with a $\mathrm{pH}_{\mathrm{i}}$ of 11.2 and an unusually high proline content $(15 \%)$. It can be divided into roughly three domains: a hydrophilic amino-terminal region (up to residue 225) containing $21 \%$ Gln (encoded by CAG), a second region of $\sim 112$ amino acids that consists essentially of apolar residues, among which proline represents $37 \%$, and a carboxy-terminal region with a more 'typical' amino acid composition containing a 'helix-turn-helix' motif.

The genomic DNA sequence corresponding to the amino-terminal region of the protein, with its high percentage of Gln, is reminiscent of the $M$ repeat in the Ubx and Antp genes (McGinnis et al. 1984b; Schneuwly et al. 1986), of the opa sequence in the Notch gene (Wharton et al. 1985), and of similar stretches in the engrailed gene (Poole et al. 1985). A corresponding cDNA fragment from $K 10$ has been used to isolate the murine opa sequence counterpart (Duboule et al. 1987). However, in contrast to the apparently randomly arranged CAG triplets interspersed with other codons that nor- mally constitute the $M$ or opa repeats so far described, the structure of the opa-like area (opla) in the K10 gene is more organized. The opla region extends from 1373 to 1539 nucleotides and is composed of seven tandemly repeated units of 24 nucleotides each. These repeated units encode the motif Gln, Gln, Gln, His, Pro, Ser, Pro, Asn, with some internal variation (Fig. 4).

Secondary structure analysis (Garnier et al. 1978) of the $K 10$ protein has shown the presence of a helix-turnhelix motif located between amino acids 390 and 418 (data not shown). Crystallographic analyses have shown the presence of such conserved helical structures in prokaryotic regulatory proteins, such as the cro and $c$ I repressor proteins of bacteriophage $\lambda$. Computer modeling studies and crystallographic data show that the conserved helical structures are the DNA-binding sites for these proteins (Pabo and Sauer 1984; Ptashne 1986; Anderson et al. 1987). Most of the amino acid residues important for the conformation of the potential DNAbinding domain structure are conserved in $K 10$, as shown by a comparison of $K 10^{\prime}$ s putative helix-turnhelix region with other potential DNA-binding domains in prokaryotes and yeast (Fig. 5). In particular, Ala, Gly, and $\mathrm{Val}$ at the respective consensus positions 5,9 , and 15 , which are known to be important for conferring the helix-turn-helix configuration necessary for interaction with the major groove of DNA, are found in K10.

It should be noted that this motif is highly divergent from the recognition helix that is found in the homeo domain (Gehring and Hiromi 1986) (Fig. 5). This result could be anticipated from the absence of a homeo box in the $K 10$ gene, as determined by cross-hybridization, even under reduced stringency conditions (McGinnis et al. 1984a,b).

Construction and expression of the $\mathrm{K} 10$ fusion protein and production of antibodies

To generate antibodies directed against the $K 10$ protein, cDNA EF43 was inserted into the $3^{\prime}$ end of the lacZ gene in the expression vector pUR288 (Rüther and MüllerHill 1983) to produce the protein in Escherichia coli. 
Prost and Deryckere et al.

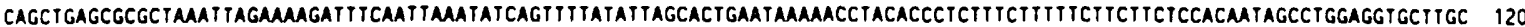
GCTGCTICTTGGAAAGGATCTICACGTGTTGCACTIGTCCACCTIMATCTTGGTGGCCCGCTICTCGGAGGGAGAACCAGAGCGTGGCCTCATCGTAGCCAACCTGGTTGCCGCCGCCAC 260

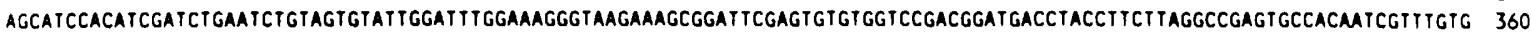

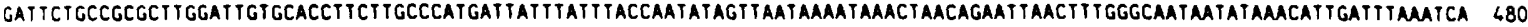
Clal

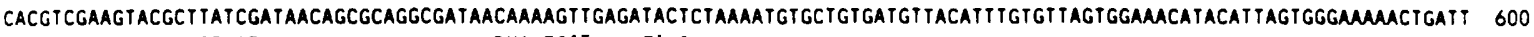
$\longrightarrow$ START CONA EG15 C Clal

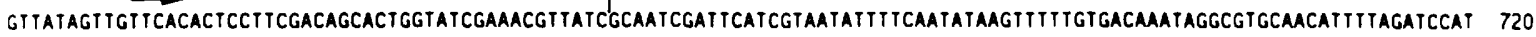

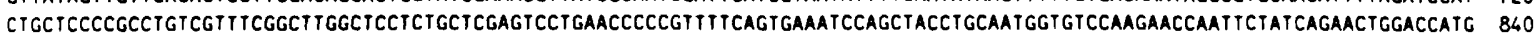
MetVal SerLySASnGInPheTYrGInAsnirpThrMet

10

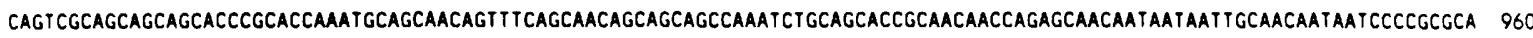

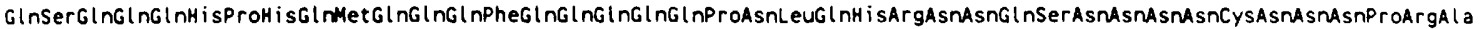
20 30 40 50

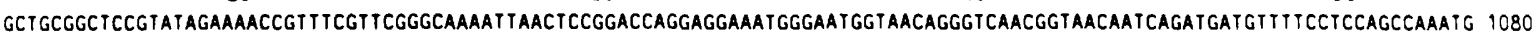

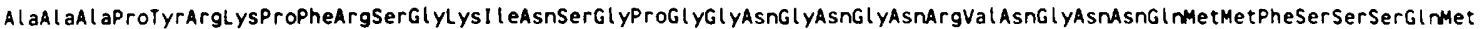
60 70 80 90

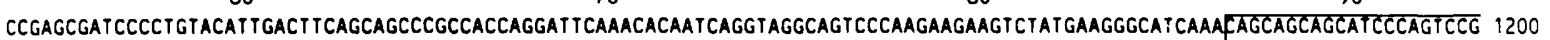

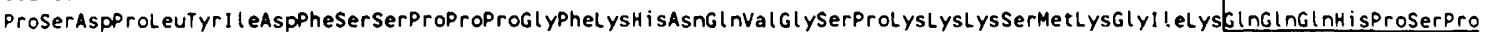
110 120 130 AACAGCAGCAGCCTCCCAGTCCGAACAGCAGCAGCATCCCAGTCCGAACAGCAGCAGCATCCCAGTCCGAACAGCAGCAGCATCCCAAT ICGAACEAGCAGCAGCATCTCAGTCCG 1320

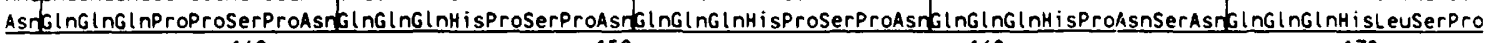
140 150 160 170

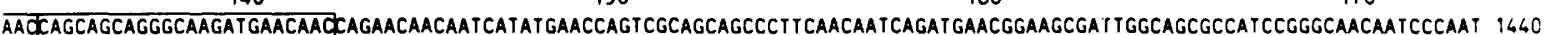

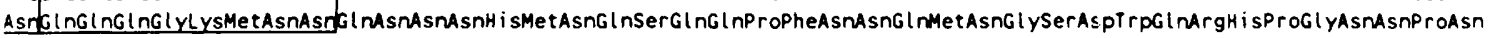
180 190 200 210 CAGATTCGCGGTGGCTTIAATGGATICCAACGCGGGCCGCCTCCGAACCGTCCTCCTCCACGCCTCATGATGGGTCCGCCAATGGGTCCGATGGGCCCCGGACCACGGGGACCCGGACCG 156O

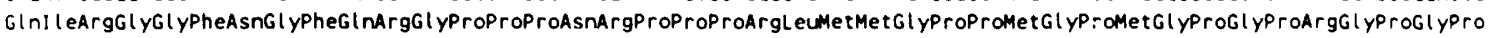
220 230 240 250 ATGGGCCCTGGAGGACCTIACCCGCAAATGCCTITCCCGCCACCGGTGCCTGGTATGCGCGGTCCCGGACACCATGGGCCCATGGTGGACCACCACCGCCCGCCGCCACCGCTCTITATG 1680

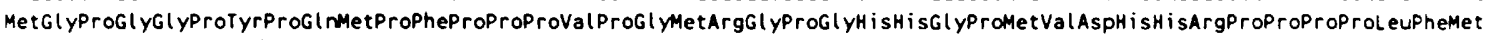
260 270 280 ECORI CDNA EF43 5 CONA E51 CGTCGCAACGGACCCGGTCCAGGTCCTATGATGGGTGICCCACCGCCCATGCACATGATGGGTCCACGGATGCCGCCGCGGGGAATICCACCGGTCGGACCTIATGGACCAATGAACATG 1800

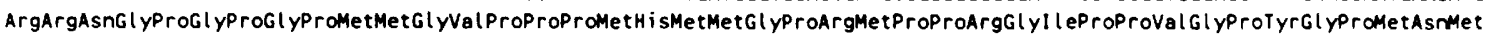
300 320 330 AATGGCGGCCGGATAATGAAGCCCAATCCCAAGCTAATCAAGCAGGTGGTGAAAGGCAAGAGCAGCATAAAGACACTAAAGAACCTGATCAATCAGTATCCCATCGAAAAGCCCTGGGTA 1920

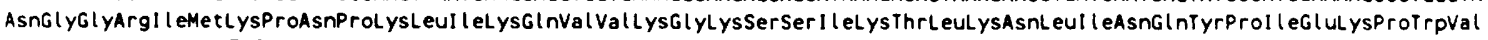
340 350 360 370

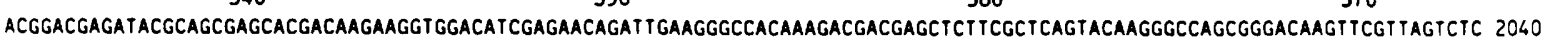

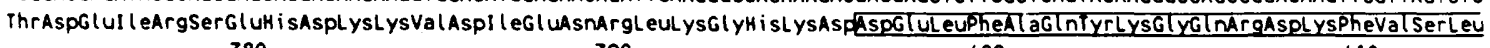
380 390 400 410 TACGAAGCGGCGCGCGAAGAGTATCTCAAGCAGgagGCAGCCACTGTCAAGGCCAAGGTGAGCATATAGAGACCCCCCAACATATCCTTATTCCCTTGGAACAGGTGTCCCATCCGAGG 2160

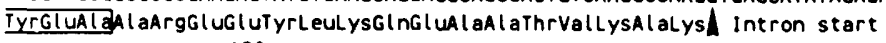
420 430

ICTAT TCAGGGAGGCACACCGATTCCGGGTACAGTTTCGGCTTAATCCGGATCACTTTGCACCCCTGATTTGATCCGTAACCCCCACCCGTCCCTCTTTGTTGCGTTTGCCGGGCGGTA 2280 GGAAAAAAATAAGGGAGCAGAGAAAGTGAAAAAGTAAGTCTCAACCTGATGCACTCTCTCGGCATTATCCTGCAGTCTGCGGAGGTTGTTGTCATATCTATTTTCACCTTCGGTTGTTC 2600 CCT TGGCACTGATATATATCCGCAAAAGAGAGAAACTATTGGCATATATTTACAACCATAGATTTATTAGGAACTGAACAAAATTGTGTAACTATTTAGAACTGCAATACAATGTGTTIA 2520 CIGCCACCTTGTATACAATTTATAAAACTAATAGAATTITGTACTGITCCGATCGTGGCAGGCGTAGTAGATTCATAATGCACTCICAGTAACTTCAATATTCTIAATTGAACGAGCCIC 26 40 AGTACTGAATCCTATGGCTGTTTICATTTTCCAATTTGTTGGCATGTGAAAAGCGAACTTAATGAATTTIAAACTGCCAGAACATCAATTTAGTTACT TAATTAGCAGATATAACCCAAC 2760 TIATGAAACTCATTGCATATGAAATGATAATAGATTIGAGTGTTAATGTTCGTGGGCGTAGTIATCTGGAAATAGCCCAAGAGAAGTTCGCAGAATGCATTTAATAGCGAAAACAGATCT 2880 GAGAGCTCTITTYACACACTCAACAACCTIGCTATTGAACACCGTIGGCAATTCTCGTATTTCTTTTGCAGGATGCCAAGTCAGACAAAGACAAAAACGCAAT TTCAAGTCAGAGCGCAG 3000 Intron end Maspal aLysSerAsplysasplysasnalalleSerSerGInSerAlaA 440

CCCCTAAGGCCGGAAGCGCTAAAGATGCAACAATICCAAATCCCTAGGCAGCCAATGCAACCGAATCCGAATACCGAATCAATCGAATCGAACCGACTAAAGAAGAGGTCGGTCGACTCA 3120 I aProLYSAL aGL YSerAL aLYSASPA L aThrI l EP ROASNPRO

450 463

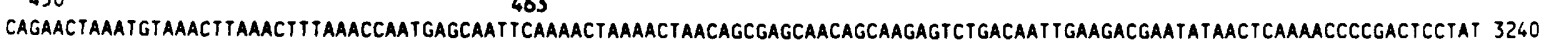
7 CONA EG15

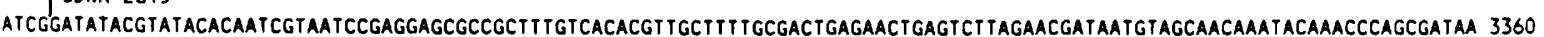
TAATTGTGGGCGAAACTCTAGTAATAGCGATAAAAATT TAGATTGCGAATCGATTCACTTGTCTAACGTAACGTACCAGTTTCCTATTCGAAAACTATT TGT TGTTCAATTCTAAGT TGC 3480 AGCCATITCAAGTATGTGTACACAATTIATGACACGCCCTTACCTATATATGTATGCATATATATIGATATATATATATATATAAATTATTTTCCTATTCGT TGCCCGAGTGCGTGGGCG 3600 CGACATAAT TGAAACAGAGTGGCCCGGATCCTAGACCTGCATAATCACACTGTAGGCCTAGATACACACTGATGTATTIAATATCTAAAACTACAACTTAGATCACTCTGTGAACGTGTG 3720

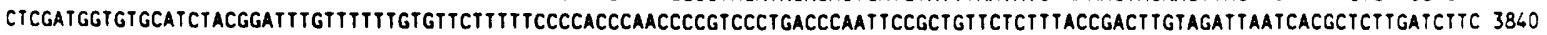
ATGACCGGATCCACCTGACAAGTAACGCCAAAGCAGCCCCACTTAGCGGATAGTAGAGCCTAGGGGACCCAACGTAAAAACTCTAGTATAGCACCAATCTCCCAACCAGTTAACATIAT 3960 ACCTAAACCCATGGTCAAGAGTAAACATTTCTGCCTT TGAAGTTGAGAACACAATTAAGCATCCCCTGGT TAAACCTGACACT TCATACTTGTTAATAGCGCCATAAACATAGCACCAAT GOBO CDNA ESI

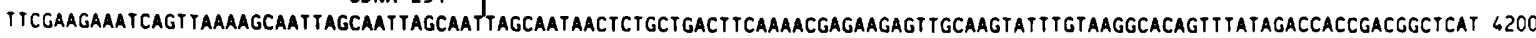
TAGGGCTCGICATGTAACTAAGCGCGGTGAAACCCAAT TGAACAGTAGTAGGTGGATIAGTTAGTIAGTCAATGGGGAGATITAACCCTCAGGTAGCAAAGTAAT TGACTGCGGGCA 6320 GAGAGTCCTAAGACTAAATAATATATTIAAAAATCTGGCCCTTIGACCTIGCTTGTCAGGTGCATITGGGTTCAATCGIAAGTTGCT TCTATATAAACACTT TCCCCATCCCCGCAATAA 6460 CDNA EF43

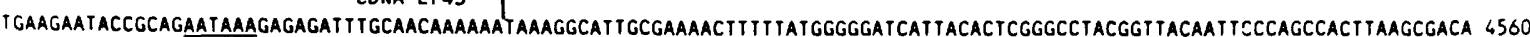
AGTITGGCCAACAATCCATCTAATAGCTAATAGCGCAATCACTGGTAATCGCAAGAGTATATAGGGCAATAGAACCCATGGATT TGACCAAAGGTAACCGAGACAATGGAGAAGCAAGAG 6680 GATITCAAACTGAACACCCACAGTGCTGTGTACTACCACTGGCGCGTITGGAGCTCACTGGCCTGATGCGCTCCTCCGGGCGTTICAAGCCTGCTTTACGTGGTATACTCCATTACGGTC 8800 AAGTTGGTGGTCACCGTGCTGITTCCCTTGAGCTTGCTGGCCAGGCTGCTGITCACCACCAACATGGCCGGATTGTGCGAGAACCTGACCATAAGTAT TACCGATATIGIGGCCAATTIG G92O AAGTITGCGATGTGTACATGGTGAGGAAGCAGCTCCATGAGATTCGCTCTCTCCTAAGGCTCATGGACGCTAGAGCCCGGCTGGTGGGCGATCCCGAGGAGATITCTGCCTTGAGGAAG 504O GAAGTGAATATCGGCACAGGGCACTTICCAGCACCTTTGGCCAGTATITICGTATTTGGCACTACTITGAGTTCGCGTCCGCGTGGTCGTTCGCCCGGATCGAGAGCTCCTGTATCCGGC S160 CTGGTICGGCGTTGACTGGATICACTCCACCAGAAACTATGIGCTCATCAATATCTACCAGCTCTTCGGCTTGATAGTGCAGGCTATACAGAACTGCGCTAGTGACTCCTATCCGCCTGC S2BO GIITCTCTGCCTGCTCACGGGTCATATGCGTGCTTTGGAGCIGAGGGTGCGGCGGATTGGCTGCAGACATATGAAGCCTGGCGGYGAGGAGGTGTACCAGGAACTCAGCGAGTGCATCCG 5LOO CGATCTGGCGCGGTCCATCGGGCTGAGGGAGACCAGCAGGCGGGCCTTTCAGTGCCCCCTITCAGTGCCCTGCATGGCCCAGTTCGTCTGCTCCGCCGCCGTCCAGTGTACCGTCGCCAT S52O

Figure 3. (See facing page for legend.) 


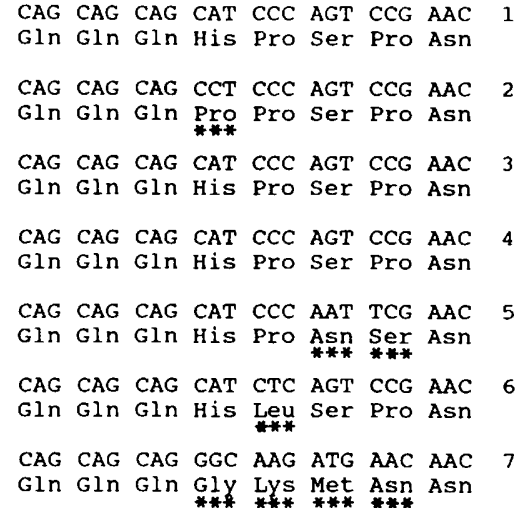

Figure 4. Nucleotide and amino acid sequence of the opla of K10. This region extends from nucleotide 1180 to 1467 on the genomic sequence. It has been aligned so as to show the seven amino acid boxes. The divergent amino acids are underlined with stars.

This cDNA clone, truncated at its internal EcoRI site during cloning, is devoid of the (CAG) rich repetitive sequences found in the $5^{\prime}$ half of the sequence. The crude protein extracts of the induced bacteria harboring the recombinant plasmids were separated on an SDS-polyacrylamide gel. As shown in Figure 6A, a fusion protein of $\sim 130 \mathrm{kD}$ was produced. This apparent molecular weight corresponds approximately to the expected molecular weight of the CDNA EF43 sequence coupled with $\beta$-galactosidase. Crude extracts containing the $\mathrm{K} 10 \mathrm{fu}$ sion protein were prepared and affinity-purified as described in Experimental procedures and were used to immunize rabbits or mice. The specificity of the antiserum obtained for the $K 10$ protein is shown by immunoblotting (Fig. 6B).

\section{The $\mathrm{K} 10$ protein is localized in the oocyte nucleus}

A short description of Drosophila melanogaster oogenesis will assist in the presentation of our results below. Detailed descriptions of oogenesis have been presented in King (1970) and in Mahowald and Kambysellis (1980).

The ovary of Drosophila consists of $\sim 17-20$ ovarioles. At the apical end of each ovariole is the germarium where free stem cells generate the cystoblasts which, by mitotic division, give rise to 16 sister cells. One of these will form the oocyte, and the other 15 will form the nurse cells. The 16 cells of germ-line origin are surrounded by a monolayer of somatically derived follicle cells, and together, the germ-line cells and the follicle cells form the egg chamber. The development of the egg chamber has been subdivided arbitrarily into 14 stages (King 1970). Each ovariole of a mature female contains six or seven egg chambers arranged in a chronological order, the youngest and smallest being in the more apical position.

From stage 7 onward, the follicle cells engage in vitellogenesis, and from stage 11 on, in the secretion of the outer protective covering of the egg, the chorion. By stage 10B, the nurse cells, which are the site for most synthesis during oogenesis, pass their nutrient-rich cytoplasm into the oocyte. In contrast, the oocyte nucleus is generally believed to be transcriptionally inactive during oogenesis (Mahowald and Kambysellis 1980).

In situ hybridization of $K 10 \mathrm{Sp} 6$ single-stranded probes to ovary sections had shown transcription of the gene early during oogenesis and detected transcripts around the oocyte nucleus (Haenlin et al. 1987). To see whether this cellular specificity could be extended to the protein, we visualized the temporal and spatial pattern of the K10 protein distribution during oogenesis by indirect immunofluorescence. The results are shown in Figure 7. The $K 10$ protein is first detected at approximately stages $8-9$ as a faint signal around the oocyte nucleus (data not shown|. This signal then intensifies, and its localization becomes nuclear, as determined by comparison with DNA-specific Hoechst staining (Fig. 7B).

The coincidence between the oocyte nucleus, as revealed by Hoechst and the $K 10$ immunofluorescence, is even more evident in some sections where the chromatin, due to a preparation artifact, appears more condensed within the nuclear membrane (Fig. 7G,H). Our observations suggest that the $K 10$ protein assumes an intranuclear localization. From stage 12 onward, i.e., when the chromosomes recondense into a karyosome (Mahowald and Kambysellis 1980), the signal disappears. The same results were observed with antisera induced in either rabbits or mice. No signal is detected when preimmune serum is used as a control, nor is a signal detected in the ovaries of females homozygous for K10. We were also unable to detect a signal on section or whole-mount embryos $(0-24 \mathrm{hr})$ or by immunoblotting extracts of early embryos. Although surprising if one considers that the $K 10$ transcripts are present at approximately the same level in early embryos and in ovaries (Haenlin et al. 1987), this result can be explained if the protein no longer assumes a specific localization.

\section{Discussion}

In an earlier report, we have shown that the $K 10$ gene is expressed in the oocyte during oogenesis (Haenlin et al. 1987). Here, we show that the $K 10$ protein is also re-

Figure 3. DNA sequence of the $K 10$ gene and corresponding amino acid sequence of the putative $K 10$ protein. The DNA sequence between the first PvulI site at the $5^{\prime}$ end (Fig. 2) and the $3^{\prime}$ end of the $K 10$ major transcript is numbered from 1 to 5520 . The amino acid sequence corresponding to the largest open reading frame is shown below the nucleotide sequence and is numbered from 1 to 463 amino acids. The boundaries of an 854 -bp intron $(2098-2952)$ are indicated by vertical arrowheads. The boundaries of the different cDNAs are also indicated. The cleavage sites of some restriction endonucleases are positioned above the nucleotide sequence. The polyadenylation signal is underlined. The sequences of the seven tandemly repeated CAG-rich boxes (1180-1347) (Fig. 4), as well as the putative DNA-binding region (1990-2049) (Fig. 6), are boxed. 


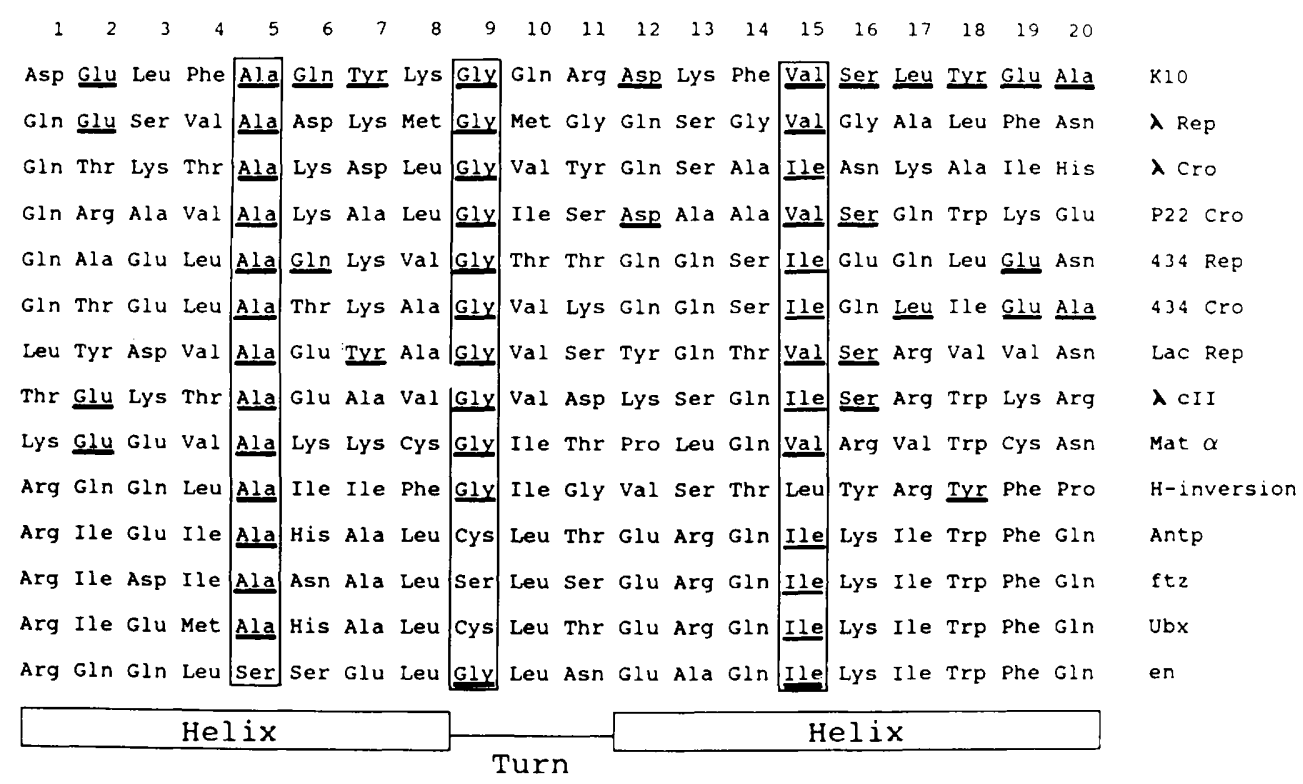

Figure 5. Amino acid sequence of $K 10$ interpreted in the helix-turn-helix model. $K 10$ sequence showing the helix-turn-helix motif was aligned with prokaryote and yeast potential DNA-binding domains (Pabo and Sauer 1984). The positions of the two helices of the DNA-binding domain are shown at the bottom. Conserved residues that appear to have an important role in maintaining the structure of the bihelical unit are boxed (Ala, Gly, Val/Ile, at positions 5, 9, and 15, respectively). Amino acids that are similar or structurally analogous to $\mathrm{K} 10$ residues are underlined. The amino acid sequences of the corresponding region of several Drosophila homeo boxes have been added for comparison. The bacterial and mat 1 sequences are from Pabo and Sauer (1984); Antp, Ubx, and $f t z$ are from McGinnis et al. (1984a); en is from Poole et al. (1985) and Fjose et al. (1985).

stricted to the oocyte, where it assumes a nuclear localization. This finding is corroborated by the presence of a helix-turn-helix motif in the sequence, which suggests a DNA-binding property for the putative $K 10$ protein. In view of this, the $K 10$ protein may have a regulatory function. The fact that this regulatory function exerts its activity on the oocyte nucleus implies that the control of expression of at least a few genes in the oocyte nucleus is essential for the establishment of dorsoventral

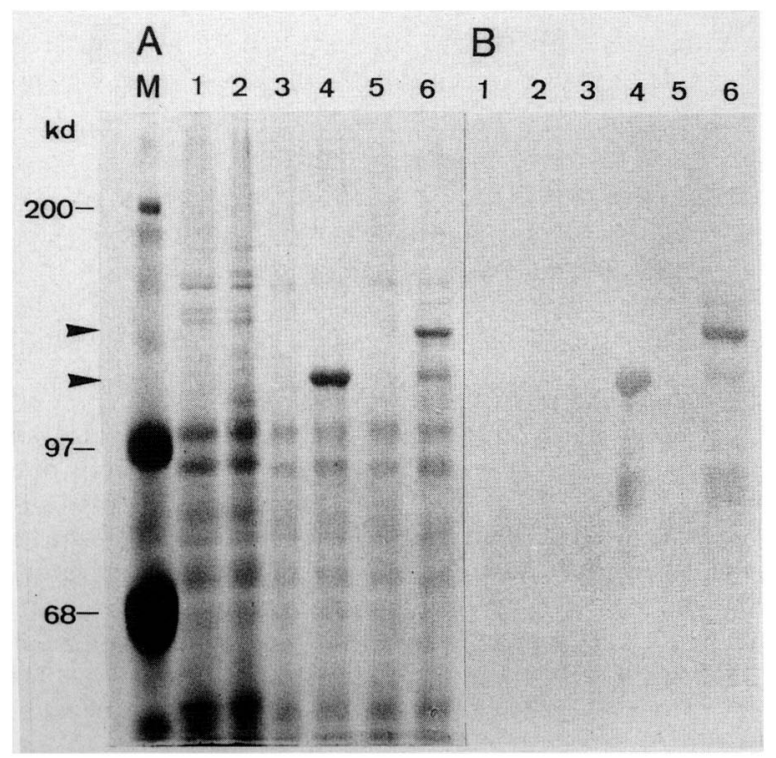

polarity of the oocyte, and possibly of the developing embryo. This result is unexpected because the oocyte nucleus, except for a short period of RNA synthesis during stage 9 , is conventionally considered to be transcriptionally inactive during oogenesis (Mahowald and Tiefert 1970).

How could our data help in understanding the mechanisms involved in the establishment of the dorsoventral polarity of the oocyte? The first manifestation of a possible dorsoventral polarity appears at approximately stage 8 , when the oocyte nucleus becomes invariably located close to the nurse cells at the future anteriordorsal region. Subsequent processes are the asymmetrical migration of the follicle cells between the oocyte and the nurse cells and, at the end of the oogenesis, the formation of the dorsally implanted appendages (Fig. 1).

The observation of dicephalic (dic) ovaries has shown that there is a strict correlation between the position of the occyte nucleus and the location of the chorion ap-

Figure 6. Expression of the $\beta$-galactosidase-K10 fusion protien in $E$. coli and production of specific antibodies. Crude protein extracts were electrophoresed on a $7 \%$ SDS-polyacrylamide gel. $(A) \mathrm{Gel}$ portion stained with silver nitrate. (Lanes 1 and 2) Noninduced and induced, respectively extracts of the bacterial strain JM103. Arrowheads indicate the induced $\beta$-galactosidase protein of $116 \mathrm{kD}$ (lane 4) vs. the noninduced protein (lane 3 ) and the $\beta$-galactosidase-K10 fusion protein of $\sim 130 \mathrm{kD}$ (lane 6 ) vs. the noninduced protein (lane 5). Protein size standards are shown in lane $M .(B)$ Gel portion transferred onto nitrocellulose (Towbin et al. 1979) and probed with crude K10 antiserum from rabbits. 
pendages (Bohrmann and Sander 1987). This correlation has been confirmed at the molecular level by an analysis of the expression of individual chorion genes by in situ hybridization. Parks and Spradling (1987) have shown that the initial spatial pattern of expression of certain chorion genes is limited to those follicle cells that are
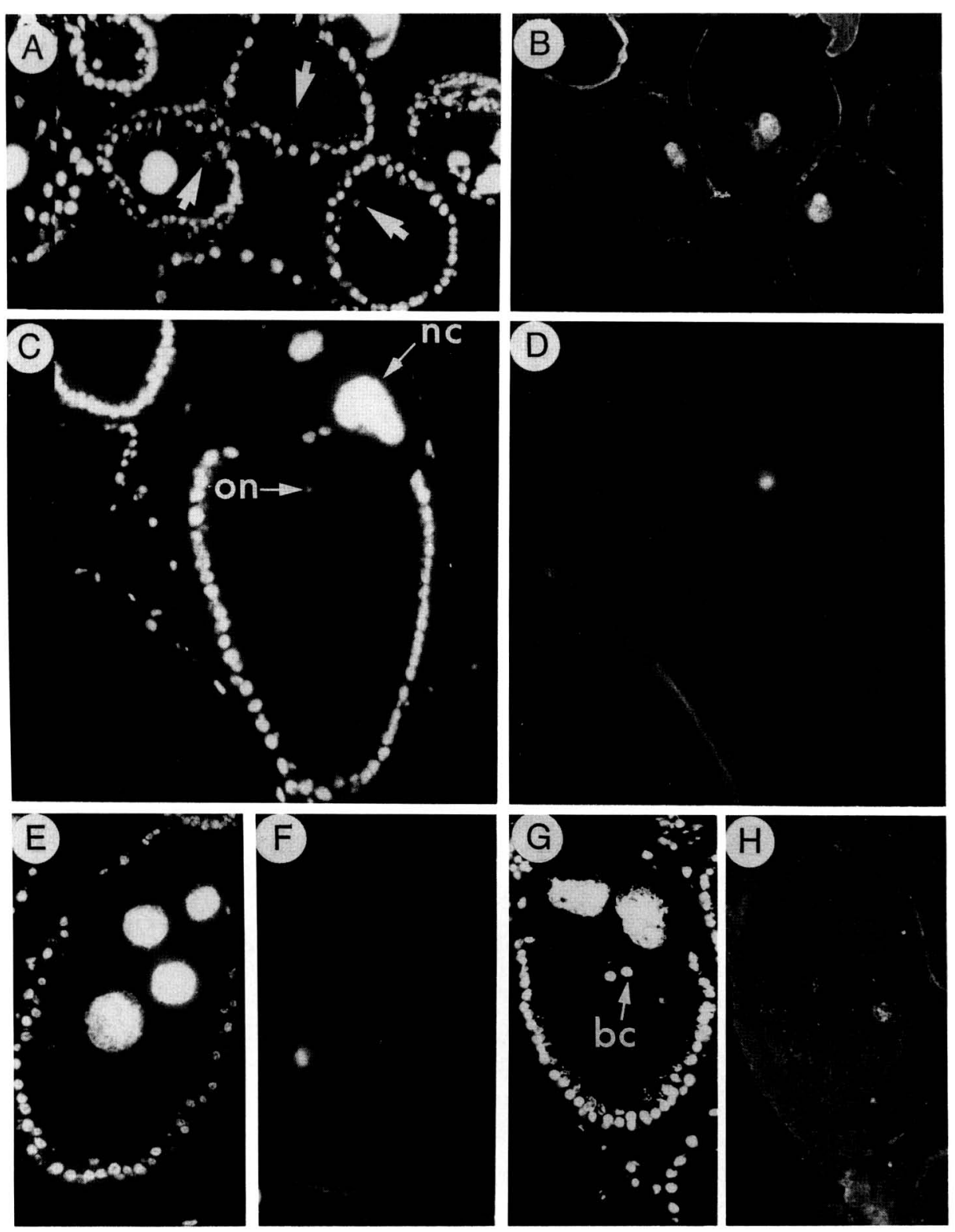

Figure 7. Localization of the $K 10$ protein on ovary sections of OreR females stained with DNA-specific Hoechst dye $(A, C, E, G)$. Egg chambers at various stages of development are visualized by the overall pattern of nuclei. The smallest nuclei belong to the follicle cells and delineate the egg chamber. The largest nuclei belong to the nurse cells (nc). An arrow indicates the oocyte nucleus (on). Also shown are border cell nuclei (bc), a group of anterior follicle cells, which attain contact with the anterior end of the oocyte after migration between the nurse cells, and are responsible for the formation of the micropyle. $(B, D, F, H)$ Corresponding staining after immunoreaction with $K 10$ antibodies, visualized by Texas red conjugated sheep anti-rabbit IgG. $(A),(B)$ Transversal sections of egg chambers of stages 8 or 9 . Two oocyte nuclei are visible with Hoechst dye, whereas the third is hardly detectable. Immunofluorescent signal clearly appears for each nucleus, indicating that at this early stage the $K 10$ product is detected both surrounding the nucleus and within the nucleus. $(C, D)$ Stage 12 egg chamber; $(E, F)$ stage $10 \mathrm{~B}$ or 11 egg chamber; $(G, H)$ stage 11 egg chamber. The high background of the immunoreaction observed in $H$ shows the organization of the wild-type egg chamber. Dorsoventral polarity can be deduced from the unequal distribution of the follicle cells, which appear thicker on the side adjacent to the oocyte nucleus and correspond to the future dorsal side of the egg. It is also visible by comparison with Hoechst staining that the chromatin occupies only a part of the nucleus, which is visualized by its perinuclear membrane visible in $H$. Superposition of both stainings clearly shows concidence of the fluorescent signal and the chromatin staining. Magnification, $175 \times$. 
close to the oocyte nucleus and will be subjected to rapid centripetal migration later on.

These observations emphasize the importance of the location of the oocyte nucleus in the morphogenetic processes that accompany the maturation of the oocyte. Together with the data obtained for $K 10$, they lead us to propose the following model to explain the establishment of the dorsoventral polarity of the oocyte and the role that $K 10$ plays in the process.

The acentric position of the oocyte nucleus appears as a prerequisite for generating the subsequent asymmetry. Microtubules, or elements of the cytoskeleton, may be required to establish and retain the nucleus in this position (Gutzeit 1986). We exclude a role for the K10 gene in the process, because the oocyte nucleus still has an acentric position in K10 ovaries (Wieschaus 1980; our own observations).

To exert its influence, the oocyte nucleus, transcriptionally inactive thus far, starts expressing genes, the products of some of which may be diffusible and capable of conveying information to the follicle cells. We assume that transcripts originating from the oocyte nucleus remain in its close vicinity and that, consequently, the product appears initially centered on the oocyte nucleus before starting to diffuse. If its diffusion is random, the follicle cells will react in accordance with their position relative to the acentric position of the oocyte nucleus, and the follicle cells closest to the oocyte nucleus will receive proportionally more of the diffusible product. This would explain the thickening of the follicular epithelium in the region close to the oocyte nucleus, where the chorionic appendages would form later on and due to a similar process. This product could function at the oocyte surface to guide follicle cell migration.

The results that we present here indicate that $K 10$ is unlikely to be such a product. Instead, its putative DNA-binding properties make it a good candidate for being one of the regulatory factors that control the expression of such a product. In accordance with our hypothesis, the alteration of the $K 10$ regulatory function by mutation would therefore result in the symmetrical migration of the anteriorly located follicle cells. This is indeed the first defect observed in the $K 10$ egg chamber (Wieschaus 1980, our own observations and legend to Fig. 1). As a consequence, all the anterior follicle cells would have the same 'dorsalized' information and would synthesize enlarged appendages encircling the anterior end of the oocyte.

What is the influence of the oocyte polarity on the subsequent development of the embryo? It can be speculated that under normal conditions, the polarity of the oocyte provides the initial trigger required for the position-dependent activation of an evenly distributed morphogen. A possible source for such a morphogen has been proposed by Anderson et al. (1985a,b) to be the Toll gene product. The activation of the Toll product, from its precursor form, is regulated in a position-dependent manner relative to the dorsoventral axis. It is possible that this activation is initiated by the inherent polarity of the oocyte.

\section{Experimental procedures \\ Indirect immunofluorescent staining of ovary sections}

Ovary sections were treated as described by Mitchison and Sedat (1983) and by White and Wilcox (1984). Ovaries of 2- to 3-day-old Oregon R or K10 homozygous females were dissected in Ringer's buffer and included in O.C.T. (Miles Laboratories). Frozen sections were dried and stored at $-80^{\circ} \mathrm{C}$. Sections were fixed in $3 \%$ paraformaldehyde in phosphate-buffered saline (PBS) for $10 \mathrm{~min}$ at room temperature, followed by extensive rinsing in PBS. After transfer in TBS [ $50 \mathrm{mM}$ Tris (pH 7.4), 150 $\mathrm{mm} \mathrm{NaCl}$ ], the slides were incubated in TBS ${ }^{*}$ [ $150 \mathrm{mM} \mathrm{NaCl}, 50$ $\mathrm{mm}$ Tris (pH 7.4), 0.2\% Nonidet P-40, 5\% sheep serum]. All successive antibody incubations and rinses were done in this buffer. Prefixed sections were then incubated with a crude antiK10 serum diluted $1 / 50$, for $3 \mathrm{hr}$ at room temperature. After several washes, the sections were further incubated in a $1 / 50$ dilution of sheep anti-rabbit IgG (Amersham). After washes in TBS and staining with DNA-specific Hoechst dye 33258 /1 $\mu \mathrm{g} / \mathrm{ml}$ ), the sections were mounted in $78 \%$ glycerol, $20 \mathrm{~mm}$ Tris (pH 7.4), and $2 \%$ propylgallate (Sigma); sealed with rubber cement to prevent dehydration, and viewed under a Zeiss microscope using epifluorescence. Control reactions with preimmune sera did not show any specific signal (data not shown).

\section{Gene fusion}

The 2.4-kb K10 cDNA EF43 had been subcloned initially into the EcoRI site of each of the three open reading frames of the pTG vectors (Courtney et al. 1984) to select the functional open reading frame on the basis of its length. The choice of the clone giving rise to the longest protein was then confirmed by in vitro expression analysis. A 1.0-kb BamHI fragment corresponding to the open reading frame of cDNA EF43 in the correct phase was subsequently isolated from pTG-K1O and inserted into the BamHI site of the pUR288 vector (Rüther and Müller-Hill 1983) to produce a $\beta$-galactosidase- $K 10$ fusion protein in $E$. coli (JM103).

\section{Expression and purification of the fusion protein}

Bacterial cultures $(700 \mathrm{ml})$ were grown in the presence of $3 \mathrm{mM}$ IPTG for $8 \mathrm{hr}$. Cells were pelleted and resuspended in $50 \mathrm{ml}$ of buffer A [20 mM Tris (pH 7.5), $10 \mathrm{mM} \mathrm{MgCl}, 0.1 \mathrm{M} \mathrm{NaCl}$ ]. The cells were lysed by sonication and spun at $12,000 \mathrm{~g}$ for $20 \mathrm{~min}$. The supernatant was adjusted to $1.6 \mathrm{M} \mathrm{NaCl}$, and $20-30 \mathrm{ml}$ was applied to a $1-\mathrm{ml} p$-amino phenyl- $\beta$-D-thiogalactosidase affinity column. Purification of the fusion protein was performed according to the technique described by Ullman (1984).

\section{Immunization of rabbits}

Rabbits were immunized by intravascular injection of a 4-ml solution containing $\mathrm{NaCl} 0.9 \%, 400 \mathrm{ng}$ of double-stranded $\operatorname{poly}(\mathrm{A})-\operatorname{poly}(\mathrm{U})$ and $15 \mu \mathrm{g}$ of purified $\beta$-galactosidase $-K 10 \mathrm{fu}-$ sion protein. They were boosted four times every 8-10 days and were bled 10 days after the last injection. Blood was obtained from the ear. Similar results were obtained with antisera induced in mice.

\section{Nucleotide sequencing}

The sequences of genomic DNA and cDNAs E51 and EF43 were determined on both strands by the dideoxynucleotide sequencing procedure (Sanger et al. 1977). Restriction fragments 
of cDNAs were inserted in M13 vectors (Messing 1983). Genomic subclones were obtained either by using known restriction sites or by generating overlapping deletions with DNase I in the presence of $\mathrm{Mn}^{2+}$ (Lin et al. 1985). The length of the open reading frames, as deduced from the sequencing data, was checked by expression in E. coli of cDNA EG15 subcloned in pUR278, pUR288, and pUR289 at the appropriate restriction sites (Rüther and Müller-Hill 1983).

\section{Acknowledgments}

We are indebted to P. Chambon, in whose laboratory this work was done, for his interest and helpful discussions. We are grateful to G. Richards, P. Simpson, and A. Nicholson for critically reading the manuscript and helping with the English. We thank the computer team, A. Eichwald, R. Fritz, D. Guinier, and J.L. Prato, for their advice during computer searches. We are grateful to M.T. Bocquel for her assistance with the immunizations. We especially thank C. Werlé for her fine graphic work, B. Boulay for the pictures, and H. Martina for patiently typing the manuscript. C.R. was the recipient of a predoctoral scholarship from the French government and a scholarship (29/269) from the Finnish Academy of Sciences. F.D. and M.H. were both funded by the Ministère de la Recherche et de l'Enseignement Supérieur. This work was supported by grants from the INSERM and CNRS.

\section{References}

Anderson, K. and C. Nüsslein-Volhard. 1984a. Genetic analysis of dorsal-ventral embryonic pattern in Drosophila. In Primers in developmental biology led. G. Malacinski and S. Bryant\}, pp. 269-289. Macmillan, New York.

- 1984b. Information for the dorsal-ventral pattern of the Drosophila embryo is stored as maternal mRNA. Nature 311: 223-227.

1986. Dorsal-group genes of Drosophila in gametogenesis and the early embryo, pp. 177-194. Alan R. Liss, New York.

Anderson, K.V., G. Jürgens, and C. Nüsslein-Volhard. 1985a. Establishment of dorsal-ventral polarity in the Drosophila embryo: Genetic studies on the role of the Toll gene product. Cell 42: 779-789.

Anderson, K.V., L. Bokla, and C. Nüsslein-Volhard. 1985b. Establishment of dorsal-ventral polarity in the Drosophila embryo: The induction of polarity by the Toll gene product. Cell 42: 791-798.

Anderson, J.E., M. Ptashne, and J.C. Harrison. 1987. Structure of the repressor-operator complex of bacteriophage 434 . $\mathrm{Na}$ ture 326: 846-852.

Bohrmann, J. and K. Sander. 1987. Aberrant oogenesis in the patterning mutant dicephalic of Drosophila melanogaster: Time-lapse recordings and volumetry in vitro. Wilhelm Roux's Arch. Dev. Biol. 196: 279-285.

Courtney, M., A. Buchwalder, L. Tessier, M. Jaye, A. Benavente, A. Balland, V. Kohl, R. Lathe, P. Tolstoshev, and J.P. Lecocq. 1984. High level production of biologically active human al-antitrypsin in E. coli. Proc. Natl. Acad. Sci. 81: 669-673.

Duboule, D., M. Haenlin, B. Galliot, and E. Mohier. 1987. DNA sequences homologous to the Drosophila opa repeat are present in murine mRNAs that are differentially expressed in fetuses and adult tissues. Mol. Cell. Biol. 7: 2003-2006.

Fjose, A., W.J. McGinnis, and W.J. Gehring. 1985. Isolation of a homeobox-containing gene from the engrailed region of Drosophila and the spatial distribution of its transcripts. Nature 313: 284-289.
Garnier, J., D.J. Osguthorpe, and B. Robson. 1978. Description of the method implemented in program Garnier. I. Mol. Biol. 120: $97-120$.

Gehring, W.J. and Y. Hiromi. 1986. Homeotic genes and the homeobox. Annu. Rev. Genet. 20: 147-173.

Gutzeit, H. 1986. The role of microtubules in the differentiation of ovarian follicles during vitellogenesis in Drosophila. Wilhelm Roux's Arch. Dev. Biol. 195: 173-181.

Haenlin, M., C. Roos, A. Cassab, and E. Mohier. 1987. Oocytespecific transcription of $f_{S}(1) K 10$ : A Drosophila gene affecting dorsal-ventral developmental polarity. EMBO $J$. 6: $801-807$.

Haenlin, M., H. Steller, V. Pirrotta, and E. Mohier. 1985. A 43 kilobase cosmid $\mathrm{P}$ transposon rescues the $f_{s}(1) \mathrm{K} 10$ morphogenetic locus and three adjacent Drosophila developmental mutants. Cell 40: 827-837.

King, R.C. 1970. Ovarian development in Drosophila melanogaster. Academic Press, New York.

Lin, H., S. Lei, and G. Wilcox. 1985. An improved DNA sequencing strategy. Anal. Biochem. 147: 114-119.

Mahowald, A.P. and M.P. Kambysellis. 1980. Oogenesis. In The genetics and biology of Drosophila. (ed. M. Ashburner and T.R.F. Wright) vol. 2D, pp. 141-224. Academic Press, London.

Mahowald, A.P. and M. Tiefert. 1970. Fine structural changes in the Drosophila oocyte nucleus during a short period of RNA synthesis. Wilhelm Roux's Arch. Dev. Biol. 165: 825.

McGinnis, W., R.L. Garber, J. Wirz, A. Kuroiwa, and W.J. Gehring. 1984a. A homologous protein-coding sequence in Drosophila homeotic genes and its conservation in other metazoans. Cell 37: 403-408.

McGinnis, W., M.S. Levine, E. Hafen, A. Kuroiwa, and W.J. Gehring. 1984b. A conserved DNA sequence in homeotic genes of the Drosophila Antennapedia and Bithorax complexes. Nature 308: 428-433.

Messing, J. 1983. New M13 vectors for cloning. Methods Enzymol. 101: 20-78.

Mitchison, T.J. and J. Sedat. 1983. Localization of antigenic determinants in whole Drosophila embryos. Dev. Biol. 99: $261-264$.

Nüsslein-Volhard, C. 1979. Maternal effect mutations that alter the spatial coordinates of the embryo of Drosophila melanogaster. In Determinants of spatial organisation led. T.S. Subtelny and I.R. Koingsbergl, pp.185-211. Academic Press, New York.

Pabo, C.O. and R.T. Sauer. 1984. Protein-DNA recognition. Annu. Rev. Biochem. 53: 293-321.

Parks, S. and A. Spradling. 1987. Spatially regulated expression of chorion genes during Drosophila oogenesis. Genes Dev. 1: 497-509.

Poole, S.J., L.M. Kauvar, B. Drees, and T. Kornberg. 1985. The engrailed locus of Drosophila: Structural analysis of an embryonic transcript. Cell 40: 37-43.

Ptashne, M. 1986. Protein-DNA interactions and gene control. In A genetic switch, pp. 33-47. Cell Press and Blackwell, Cambridge, Massachussets.

Rüther, V. and B. Müller-Hill. 1983. Easy identification of cDNA clones. EMBO J. 2: 1791-1794.

Sanger, F., S. Nicklen, and A.R. Coulson. 1977. DNA sequencing with chain-terminating inhibitors. Proc. Natl. Acad. Sci. 74: 5463-5467.

Schneuwly, S., A. Kuroiwa, P. Baumgartner, and W.J. Gehring. 1986. Structural organization and sequence of the homeotic gene Antennapedia of Drosophila melanogaster. EMBO J. 5: $733-739$.

Schupbach, T. 1987. Germ line and soma cooperate during oo- 
genesis to establish the dorsoventral pattern of egg shell and embryo in Drosophila melanogaster. Cell 49: 699-707.

Towbin, H., T. Staehelin, and J. Gordon. 1979. Electrophorectic transfer of proteins from polyacrylamide gels to nitrocellulose sheets: Procedure and some applications. Proc. Natl. Acad. Sci. 76: 4350-4354.

Ullman, A. 1984. One step purification of hybrid proteins which have $\beta$-galactosidase activity. Gene 29: 27-31.

Wharton, K.A., B. Yedvobnick, V.G. Finnerty, and S. ArtavanisTsakonas. 1985. opa: A novel family of transcribed repeats shared by the Notch locus and other developmentally regulated loci in D. melanogaster. Cell 40: 55-62.

White, R.A. and M. Wilcox. 1984. Protein products of the Bithorax complex in Drosophila. Cell 39: 163-171.

Wieschaus, E. 1979. $f_{s}(1) K 10$, a female sterile mutation altering the pattern of both the egg coverings and the resultant embryos in Drosophila. In Cell lineage, stem cell and celi differentiation (ed. N. le Douarin), pp. 291-302. Elsevier/ North-Holland Biomedical Press, New York.

1980. A combined genetic and mosaic approach to the study of oogenesis in Drosophila. In Development and neurobiology of Drosophila (ed. O. Siddiqi, P. Babu, L.M. Hall, and J.C. Hall), pp. 85-94. Plenum Press, New York.

Wieschaus, E., J.L. Marsh, and W. Gehring. 1978. fs(1)K10, a germline-dependent female sterile mutation causing abnormal chorion morphology in Drosophila melanogaster. Wilhelm Roux's Arch. Dev. Biol. 184: 75-82. 


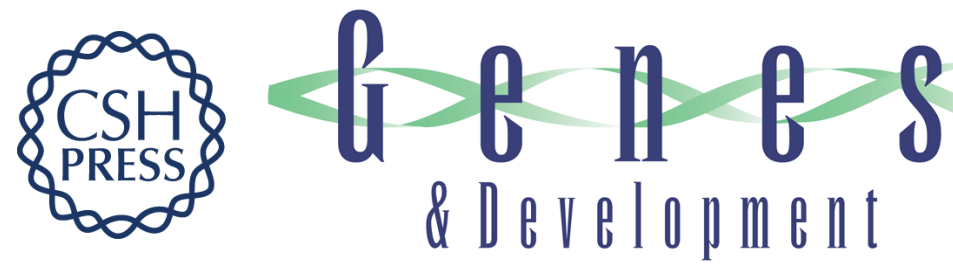

\section{Role of the oocyte nucleus in determination of the dorsoventral polarity of Drosophila as revealed by molecular analysis of the $\mathrm{K} 10$ gene}

Edouard Prost, François Deryckere, Christophe Roos, et al.

Genes Dev. 1988, 2:

Access the most recent version at doi:10.1101/gad.2.7.891

\section{License}

Email Alerting Receive free email alerts when new articles cite this article - sign up in the box at the top Service right corner of the article or click here.

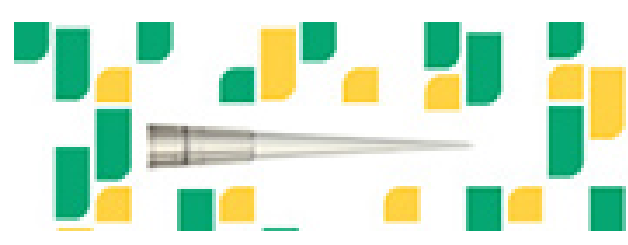

Focused on your science. 